\title{
Synthesis, Properties and Crystal Structures of Iron-(II) and -(III) Complexes of $2,2^{\prime}: 6^{\prime}, 2^{\prime \prime}: 6^{\prime \prime}, 2^{\prime \prime \prime}$-Quaterpyridine $\dagger$
}

\author{
Chi-Ming Che, ${ }^{*}, a$ Chin-Wing Chan, ${ }^{a}$ San-Ming Yang, ${ }^{a}$ Chun-Xiao Guo, ${ }^{a}$ Chung-Yuan Lee ${ }^{b}$ and \\ Shie-Ming Peng ${ }^{b}$ \\ a Department of Chemistry, The University of Hong Kong, Pokfulam Road, Hong Kong \\ ${ }^{b}$ Department of Chemistry, National Taiwan University, Taipei, Taiwan
}

\begin{abstract}
Several iron complexes of $2,2^{\prime}: 6^{\prime}, 2^{\prime \prime}: 6^{\prime \prime}, 2^{\prime \prime \prime}$-quaterpyridine $(L)$ in di- and tri-valent oxidation states have been prepared, and the structures of $\left[\mathrm{FeL}\left(\mathrm{OH}_{2}\right)_{2}\right]\left[\mathrm{ClO}_{4}\right]_{2}$, $\left[\left\{\mathrm{FeL}\left(\mathrm{ClO}_{4}\right)\right\}_{2}(\mu-\mathrm{O})\right] \cdot 8.5 \mathrm{H}_{2} \mathrm{O} 2 \cdot 8.5 \mathrm{H}_{2} \mathrm{O}$ and $\left[\{\mathrm{FeL}(\mathrm{Cl})\}_{2}(\mu-\mathrm{O})\right]\left[\mathrm{ClO}_{4}\right]_{2} \cdot 2 \mathrm{H}_{2} \mathrm{O} 3 \cdot 2 \mathrm{H}_{2} \mathrm{O}$, have been determined by $\mathrm{X}$-ray crystal analyses. In each complex, the quaterpyridine ligand is planar. In 2, the $\mathrm{Fe}^{\mathrm{II}}$ is seven-co-ordinate with the $\left[\mathrm{ClO}_{4}\right]$ acting as a bidentate ligand with $\mathrm{Fe}-\mathrm{O}-\mathrm{Fe} 155.2(4)^{\circ}$. In 3 , the $\mathrm{Fe}-\mathrm{O}-\mathrm{Fe}$ unit is linear and the $\mathrm{Fe}-\mathrm{O}$ distance of $1.779(1) \AA$ is considerably shorter than the $\mathrm{Fe}-\mathrm{O}\left(\mathrm{OH}_{2}\right)$ distance of $2.141(5) \AA$ in 1 . The results of molecular orbital calculations revealed that the unoccupied molecular orbitals of 2 and 3 mainly comprise the $\pi^{*}$ orbital of $\mathrm{L}$. In 2 , bending of the $\mathrm{Fe}-\mathrm{O}-\mathrm{Fe}$ unit gives rise to a decrease in the antibonding interaction between $\mathrm{Fe}\left(\mathrm{d}_{y_{2}}\right)$ and $O\left(\mathrm{p}_{y}\right)$ orbitals. Complex 3 is less stable in the spinpaired $(S=0)$ than in open-shell $(S=1)$ state by $49.3 \mathrm{kcal} \mathrm{mol}^{-1}$. This coincides with magnetic susceptibility measurements which show that 3 is paramagnetic with $\mu_{\text {eff }} 1.75 \mu_{\mathrm{B}}$ per iron atom.
\end{abstract}

Polypyridines are among the most common chelating ligands for transition-metal complexes of photochemical and electrochemical interests. ${ }^{\prime}$ Although the co-ordination chemistry of $2,2^{\prime}$-bipyridine and $2,2^{\prime}: 6^{\prime}, 2^{\prime \prime}$-terpyridine are well documented, ${ }^{2}$ related studies on oligomeric pyridines are still in the development stage. Previous studies by Constable et al. ${ }^{3}$ and Che and co-workers ${ }^{4}$ have demonstrated the versatile coordinating ability of $2,2^{\prime}: 6^{\prime}, 2^{\prime \prime}: 6^{\prime \prime}, 2^{\prime \prime \prime}$-quaterpyridine $(\mathrm{L})$, which usually functions as a tetradentate chelating ligand. Iron complexes of quaterpyridine have also been studied extensively by Pispisa and co-workers ${ }^{5}$ but their crystal structures have not been reported. Our interests in iron-quaterpyridine complexes stem from previous studies indicating that high-valent $\mathrm{Fe}=\mathrm{O}$ species are likely to be generated through oxidation of $\mathrm{Fe}^{I I}$ and/or $\mathrm{Fe}^{\mathrm{III}}$ by an oxygen atom donor such as $\mathrm{H}_{2} \mathrm{O}_{2}$ or $\mathrm{Bu}^{\mathrm{t}} \mathrm{O}_{2} \mathrm{H}$ in the presence of pyridyl ligands. ${ }^{6}$ As quaterpyridine is a good chelating ligand, it may be useful for the study of high-valent $\mathrm{Fe}=\mathrm{O}$ chemistry. In this work, the synthesis and crystal structures of several tetradentate, quaterpyridine iron complexes are reported.

\section{Experimental}

Materials. - 2,2': $6^{\prime}, 2^{\prime \prime}: 6^{\prime \prime}, 2^{\prime \prime \prime}$-Quaterpyridine was prepared by literature methods; ${ }^{3 a} \mathrm{FeCl}_{3}, \mathrm{Fe}\left(\mathrm{ClO}_{4}\right)_{2} \cdot x \mathrm{H}_{2} \mathrm{O}$ and $\mathrm{Fe}$ $\left(\mathrm{ClO}_{4}\right)_{3} \cdot x \mathrm{H}_{2} \mathrm{O}$ were used as received. Acetonitrile was distilled over $\mathrm{KMnO}_{4}$ followed by $\mathrm{CaH}_{2}$.

Physical Measurements and Instrumentation.-Infrared spectra were measured on a Nicolet 20 SXC FT-IR spectrometer, and UV/VIS spectra on a Milton Roy Spectronic 3000 spectrophotometer. Elemental analyses were performed at the Shanghai Institute of Organic Chemistry, Chinese Academy of Science and at the Department of Chemistry of National Taiwan University.

† Supplementary data available: see Instructions for Authors, J. Chem. Soc., Dalton Trans., 1995, Issue 1, pp. xxv-xxx.

Non-SI units employed: $\mu_{\mathrm{B}} \approx 9.27402 \times 10^{-24} \mathrm{~J} \mathrm{~T}^{-1}, \mathrm{cal} \approx 4.184 \mathrm{~J}$.
$X$-Ray Crystal Structure Determination.-Crystal data are listed in Table 1. The diffraction data were collected on an Enraf-Nonius CAD4 diffractometer at $298 \mathrm{~K}$ (graphitemonochromated Mo-K $\alpha$ radiation, $\lambda=0.71070 \AA$ ) with the $\theta-2 \theta$ method. The unit-cell dimensions were obtained from a least-squares fit of 25 reflections in the range of $18.56<2 \theta<28.10$ and $18.82<2 \theta<27.98^{\circ}$ for 1 and 2 respectively, and 24 reflections in the range of $18.58<2 \theta<24.02^{\circ}$ for 3 . Calculations were carried out with a NRCVAX refinement program. ${ }^{7}$ The final agreement factors $R\left[=\left(\Sigma\left|F_{\mathrm{o}}-F_{\mathrm{c}}\right|\right) / \Sigma\left|F_{\mathrm{o}}\right|\right], R^{\prime}\left\{=\left[\Sigma w\left(\left|F_{\mathrm{o}}\right|-\left|F_{\mathrm{c}}\right|\right)^{2} / \Sigma w\left|F_{\mathrm{o}}\right|_{2}\right]^{\frac{1}{2}}\right\}$ and $S\left\{=\left[\Sigma w\left(\left|F_{0}-F_{\mathrm{c}}\right|\right)^{2} /(n-p)\right]^{\frac{1}{2}}\right\}$ are also listed in Table 1 . The atomic coordinates of all non-hydrogen atoms of 1,2 and 3 are listed in Tables 2, 3 and 4 respectively, and selected bond distances and angles are given in Table 5 .

Additional material available from the Cambridge Crystallographic Data Centre comprises $\mathrm{H}$-atom coordinates, thermal parameters and remaining bond lengths and angles.

Preparations. - $\left[\mathrm{FeL}\left(\mathrm{OH}_{2}\right)_{2}\right]\left[\mathrm{ClO}_{4}\right]_{2}$ 1. A mixture of Fe$\left(\mathrm{ClO}_{4}\right)_{2} \cdot x \mathrm{H}_{2} \mathrm{O}(0.1 \mathrm{~g})$ and $\mathrm{L}(46.5 \mathrm{mg}, 0.15 \mathrm{mmol})$ in acetonitrile $\left(50 \mathrm{~cm}^{3}\right)$ was heated at $70^{\circ} \mathrm{C}$ for $15 \mathrm{~min}$. The solution was filtered and concentrated to about $10 \mathrm{~cm}^{3}$. Upon diffusion of diethyl ether into the solution, red needles were obtained in $87 \%$ yield (Found: $\mathrm{C}, 37.8 ; \mathrm{H}, 3.1 ; \mathrm{N}, 8.3$. Calc. for $\mathrm{C}_{20} \mathrm{H}_{18} \mathrm{Cl}_{2} \mathrm{FeN}_{4} \mathrm{O}_{10}$ : C, 37.15; $\left.\mathrm{H}, 2.80 ; \mathrm{N}, 8.65\right)$. IR $\left(\mathrm{cm}^{-1}\right)$ : $1463 \mathrm{~m}, 1143 \mathrm{~s}, 1111 \mathrm{~s}, 1087 \mathrm{~s}, 775 \mathrm{~m}, 627 \mathrm{~m}$. UV/VIS in $\mathrm{CH}_{3} \mathrm{CN}:\left[\lambda_{\max } / \mathrm{nm}\left(10^{-3} \varepsilon / \mathrm{dm}^{3} \mathrm{~mol}^{-1} \mathrm{~cm}^{-1}\right)\right]: 232$ (35), 293 $(18), 301$ (22), 338 (12), 365 (2.9), 515 (0.72); $\mu_{\text {eff }}=$ $0 \mu_{\mathrm{B}}$ (Evans' method).

$\left[\left\{\mathrm{FeL}\left(\mathrm{ClO}_{4}\right)\right\}_{2}(\mu-\mathrm{O})\right]$ 2. A mixture of $\mathrm{Fe}\left(\mathrm{ClO}_{4}\right)_{3} \cdot x \mathrm{H}_{2} \mathrm{O}(0.2$ g) and $\mathrm{L}(0.05 \mathrm{~g}, 16 \mathrm{mmol})$ in water-ethanol $\left(20 \mathrm{~cm}^{3}, 10: 1 \mathrm{v} / \mathrm{v}\right)$ was stirred at room temperature for $1 \mathrm{~d}$. Upon standing for several days, reddish brown crystals were obtained, yield $15 \%$ (Found: C, 49.9; $\mathrm{H}, 3.1 ; \mathrm{N}, 11.40$. Calc. for $\mathrm{C}_{40} \mathrm{H}_{28} \mathrm{Cl}_{2} \mathrm{Fe}_{2} \mathrm{~N}_{8}$ $\mathrm{O}_{9}: \mathrm{C}, 50.7 ; \mathrm{H}, 2.95 ; \mathrm{N}, 11.85 \%$ ); $\mu_{\text {eff }}=0 \mu_{\mathrm{B}}$ (Evans' method).

$\left[\{\mathrm{FeL}(\mathrm{Cl})\}_{2}(\mu-\mathrm{O})\right]\left[\mathrm{ClO}_{4}\right]_{2}$ 3. $2,2^{\prime}: 6^{\prime}, 2^{\prime \prime}: 6^{\prime \prime}, 2^{\prime \prime \prime}$-Quaterpyridine $(0.025 \mathrm{~g})$ in $\mathrm{CH}_{2} \mathrm{Cl}_{2}\left(25 \mathrm{~cm}^{3}\right)$ was added to an ethanolic solution of $\mathrm{FeCl}_{3}\left(0.013 \mathrm{~g}\right.$ in $\left.10 \mathrm{~cm}^{3}\right)$. The mixture was refluxed for $2 \mathrm{~d}$. Upon additon of $\mathrm{LiClO}_{4}$ to the filtrate, a yellow-orange 
Table 1 Crystallographic data

$\begin{array}{ll}\text { Complex } & \mathbf{1} \\ \text { Formula } & \mathrm{C}_{20} \mathrm{H}_{18} \mathrm{Cl}_{2} \mathrm{FeN}_{4} \mathrm{O}_{10} \\ M & 601.28 \\ \text { Crystal dimensions/mm } & 0.30 \times 0.35 \times 0.50 \\ \text { Crystal system } & \text { Monoclinic } \\ \text { Space group } & C 2 / c \\ a / \AA & 14.633(5) \\ b / \AA & 11.134(4) \\ c / \AA & 15.123(6) \\ \beta / /^{\circ} & 98.84(3) \\ U / \AA^{3} & 2435(2) \\ F(000) & 1224 \\ Z & 4 \\ D \text { c/g cm } & 1.64 \\ \mu / \mathrm{cm}^{-1} & 8.99 \\ 2 \theta_{\text {max }}{ }^{\circ} & 50 \\ h, k, l & -17 \text { to } 17,0-13,0-17 \\ \text { Unique data measured } & 2129 \\ \text { Unique data used, } n & 1617\left[I_{\mathrm{o}} \geqslant 2 \sigma\left(I_{\mathrm{o}}\right)\right] \\ \text { Number of variables, } p & 169 \\ \text { Number of atoms } & 28 \\ R, R^{\prime} \text { and } S & 0.063,0.060,1.76 \\ \text { Weighting scheme } & \text { Unit weight } \\ \rho / \mathrm{e} \AA^{-3} & -0.54 \text { to }+0.67\end{array}$

$2 \cdot 8.5 \mathrm{H}_{2} \mathrm{O}$
$\mathrm{C}_{40} \mathrm{HCl}_{2} \mathrm{Fe}_{2} \mathrm{~N}_{8} \mathrm{O}_{17.5}$
1100.34
$0.20 \times 0.20 \times 0.25$
Monoclinic
$P 2 / c$
$11.420(2)$
$22.046(4)$
$18.348(4)$
$97.82(2)$
$4576(2)$
1134
2
1.57
8.16
45
-12 to $12,0-23,0-19$
5970
$2784\left[I_{\mathrm{o}} \geqslant 2 \sigma\left(I_{\mathrm{o}}\right)\right]$
629
97.5
$0.063,0.058,2.50$
$\left[1 / \sigma\left(F_{\mathrm{o}}\right)\right]^{2}$
-0.44 to +0.62

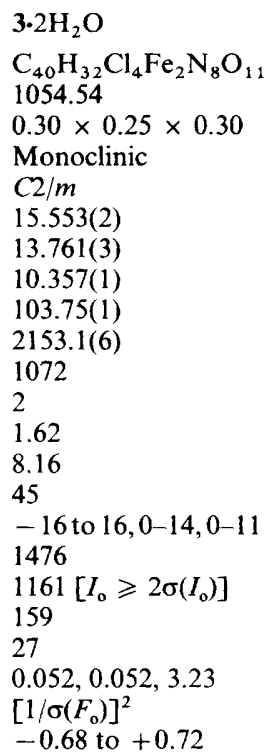

Table 2 Non-hydrogen atomic coordinates with estimated standard deviations (e.s.d.s) for $\left[\mathrm{FeL}\left(\mathrm{OH}_{2}\right)_{2}\right]\left[\mathrm{ClO}_{4}\right]$

\begin{tabular}{llll} 
Atom & $x$ & $y$ & $z$ \\
$\mathrm{Fe}$ & 0 & $0.08208(12)$ & \multicolumn{1}{l}{$1 / 4$} \\
$\mathrm{~N}(1)$ & $-0.0657(4)$ & $0.1519(5)$ & $0.1196(3)$ \\
$\mathrm{N}(2)$ & $-0.0414(4)$ & $-0.0722(5)$ & $0.1695(3)$ \\
$\mathrm{C}(1)$ & $-0.0737(5)$ & $0.2677(7)$ & $0.0979(5)$ \\
$\mathrm{C}(2)$ & $-0.1141(5)$ & $0.3071(7)$ & $0.0146(5)$ \\
$\mathrm{C}(3)$ & $-0.1468(5)$ & $0.2229(8)$ & $-0.0483(5)$ \\
$\mathrm{C}(4)$ & $-0.1388(5)$ & $0.1030(7)$ & $-0.0280(4)$ \\
$\mathrm{C}(5)$ & $-0.0979(4)$ & $0.0688(6)$ & $0.0574(4)$ \\
$\mathrm{C}(6)$ & $-0.0867(4)$ & $-0.0577(6)$ & $0.0862(4)$ \\
$\mathrm{C}(7)$ & $-0.1190(6)$ & $-0.1562(7)$ & $0.0344(5)$ \\
$\mathrm{C}(8)$ & $-0.1034(6)$ & $-0.2688(7)$ & $0.0702(5)$ \\
$\mathrm{C}(9)$ & $-0.0562(6)$ & $-0.2845(7)$ & $0.1553(5)$ \\
$\mathrm{C}(10)$ & $-0.0257(5)$ & $-0.1821(6)$ & $0.2035(4)$ \\
$\mathrm{O}(1)$ & $0.1289(3)$ & $0.1116(4)$ & $0.2031(3)$ \\
$\mathrm{O}(2)$ & $0.6700(10)$ & $-0.1571(10)$ & $0.1438(9)$ \\
$\mathrm{O}(3)$ & $0.5907(7)$ & $-0.0412(12)$ & $0.2015(11)$ \\
$\mathrm{O}(4)$ & $0.7239(10)$ & $-0.0931(15)$ & $0.2685(8)$ \\
$\mathrm{O}(5)$ & $0.7217(6)$ & $0.0288(8)$ & $0.1618(6)$ \\
$\mathrm{C} 1$ & $0.67524(14)$ & $-0.06192(18)$ & $0.19430(13)$ \\
& & &
\end{tabular}

$\mathrm{mol}^{-1}$ ). The corrected molar susceptibility of $3, \chi_{m}^{\prime}=$ $2.5520 \times 10^{-3} \mathrm{~cm}^{3} \mathrm{~g}^{-1}$ and thus the effective magnetic moment is $1.75 \mu_{\mathrm{B}}$ per iron atom.

Molecular Orbital Calculations.-Molecular orbital calculations on 2 and 3 were performed by the complete neglect of differential overlaps (CNDO) method. The parameters used for iron were taken from Clark and Allen, ${ }^{9}$ those for other atoms are from Pople and Beveridge. ${ }^{10}$ The geometric factors were obtained from the X-ray diffraction studies. The atomic positions were transformed into Cartesian coordinates by taking oxygen atom as the origin $(0,0,0)$ with the $x-, y$ - and $z$ directions defined as follows: both the $y$ - and $z$-axis are on the imaginary plane containing the $\mathrm{Fe}-\mathrm{O}-\mathrm{Fe}$ moiety. The $\mathrm{Fe}-\mathrm{O}$ bond is taken as the $z$-axis in 3 ; for 2 , the $z$-axis is parallel to the line joining the two $\mathrm{Fe}$ atoms. The near frontier orbital energies and compositions of $\mathbf{2}$ and $\mathbf{3}$ are summarized in Tables 6 and 7 respectively.

\section{Results and Discussion}

The reaction of $\mathrm{FeSO}_{4}$ with $\mathrm{L}$ and some of the oxidation chemistry of $\mathrm{Fe}^{\mathrm{II}}-\mathrm{L}$ complexes have been extensively studied by Pispisa and co-workers, ${ }^{5}$ but no structural study has been reported. In this work, $\left[\mathrm{FeL}\left(\mathrm{OH}_{2}\right)_{2}\right]^{2+} 1$ was prepared by treating $\mathrm{Fe}\left(\mathrm{ClO}_{4}\right)_{2} \cdot x \mathrm{H}_{2} \mathrm{O}$ with $\mathrm{L}$ in boiling acetonitrile, and isolated as a red perchlorate salt. Its UV/VIS absorption spectrum displays an intense absorption around $300 \mathrm{~nm}$ ( $\varepsilon>10^{4} \mathrm{dm}^{3} \mathrm{~mol}^{-1} \mathrm{~cm}^{-1}$ ), which is intraligand in nature. There is also an intense broad absorption band ranging from 400 to $500 \mathrm{~nm}\left(\varepsilon \approx 10^{3} \mathrm{dm}^{3} \mathrm{~mol}^{1} \mathrm{~cm}^{-1}\right)$, which is due to a Fe ${ }^{\mathrm{II}} \longrightarrow \mathrm{L}$ charge-transfer transition

The complex gradually undergoes ligand dissociation in acidic or basic solution. However, it is more stable in degassed citrate and/or phosphate buffer solutions $\left(\approx 0.1 \mathrm{~mol} \mathrm{dm}^{-3}\right)$. Our attempt to prepare $\left[\mathrm{FeL}\left(\mathrm{OH}_{2}\right)(\mathrm{OH})\right]^{2+}$ by treating $\mathrm{Fe}\left(\mathrm{ClO}_{4}\right)_{3}$. $x \mathrm{H}_{2} \mathrm{O}$ with $0.1 \mathrm{~mol} \mathrm{dm}^{-3} \mathrm{~L}$ in ethanol-water was unsuccessful. Instead, a seven-co-ordinated dinuclear $\mathrm{Fe}^{\mathrm{II}}$ complex, 2, was formed. Presumably, ethanol acts as the reductant for the reduction of $\mathrm{Fe}^{\mathrm{III}}$ to $\mathrm{Fe}^{\mathrm{II}}$. In aqueous solutions, complex 2 easily converts to 1 . In fact, the cyclic voltammograms of $\mathbf{1}$ and 2 in $0.1 \mathrm{~mol} \mathrm{dm}^{-3} \mathrm{CF}_{3} \mathrm{SO}_{3} \mathrm{H}$ are virtually identical. In alcoholic solution and with $\mathrm{FeCl}_{3}$ as the starting material, the yelloworange $\left[\mathrm{FeL}(\mathrm{Cl})_{2}\right] \mathrm{ClO}_{4}$ complex was obtained as the initial 
Table 3 Non-hydrogen atomic coordinates with e.s.d.s for $\left[\left\{\mathrm{FeL}\left(\mathrm{ClO}_{4}\right)\right\}_{2}(\mu-\mathrm{O})\right]$

\begin{tabular}{|c|c|c|c|c|c|c|c|}
\hline Atom & $x$ & $y$ & $z$ & Atom & $x$ & $y$ & $z$ \\
\hline $\mathrm{Fe}(1)$ & $0.95123(16)$ & $0.65920(9)$ & $0.11035(10)$ & $C(15)$ & $0.7996(12)$ & $0.7527(7)$ & $0.1859(7)$ \\
\hline $\mathrm{Fe}(2)$ & $0.71752(15)$ & $0.66948(8)$ & $-0.03369(10)$ & $C(16)$ & $0.7550(12)$ & $0.6955(6)$ & $0.2097(8)$ \\
\hline $\mathrm{Cl}(1)$ & $1.0945(4)$ & $0.60192(20)$ & $0.22362(22)$ & $\mathrm{C}(17)$ & $0.6633(12)$ & $0.6922(9)$ & $0.2535(7)$ \\
\hline $\mathrm{Cl}(2)$ & $0.5603(3)$ & $0.62395(16)$ & $-0.14920(19)$ & $\mathrm{C}(18)$ & $0.6251(13)$ & $0.6384(10)$ & $0.2712(9)$ \\
\hline $\mathrm{O}$ & $0.8332(7)$ & $0.6469(3)$ & $0.0365(4)$ & $C(19)$ & $0.6791(12)$ & $0.5859(9)$ & $0.2475(8)$ \\
\hline$O(1)$ & $1.0010(8)$ & $0.5749(4)$ & $0.1659(5)$ & $C(20)$ & $0.7711(12)$ & $0.5950(7)$ & $0.2068(8)$ \\
\hline$O(2)$ & $1.0845(8)$ & $0.6690(4)$ & $0.2039(5)$ & $\mathrm{C}(21)$ & $0.8784(11)$ & $0.5985(6)$ & $-0.1352(7)$ \\
\hline $\mathrm{O}(3)$ & $1.0658(10)$ & $0.5895(5)$ & $0.2983(5)$ & $C(22)$ & $0.9744(12)$ & $0.5877(6)$ & $-0.1742(8)$ \\
\hline $\mathrm{O}(4)$ & $1.2148(8)$ & $0.5804(5)$ & $0.2179(6)$ & $\mathrm{C}(23)$ & $1.0401(12)$ & $0.6347(7)$ & $-0.1910(8)$ \\
\hline$O(5)$ & $0.6470(7)$ & $0.5915(4)$ & $-0.0951(4)$ & $\mathrm{C}(24)$ & $1.0101(12)$ & $0.6921(7)$ & $-0.1702(7)$ \\
\hline $\mathrm{O}(6)$ & $0.5839(7)$ & $0.6892(4)$ & $-0.1273(5)$ & $C(25)$ & $0.9135(11)$ & $0.6999(6)$ & $-0.1306(6)$ \\
\hline$O(7)$ & $0.5849(8)$ & $0.6140(4)$ & $-0.2242(5)$ & $\mathrm{C}(26)$ & $0.8783(10)$ & $0.7591(6)$ & $-0.1050(6)$ \\
\hline $\mathrm{O}(8)$ & $0.4384(7)$ & $0.6073(4)$ & $-0.1421(5)$ & $\mathrm{C}(27)$ & $0.9242(12)$ & $0.8139(6)$ & $-0.1246(7)$ \\
\hline$N(1)$ & $1.0844(8)$ & $0.6154(4)$ & $0.0420(5)$ & $C(28)$ & $0.8794(13)$ & $0.8672(6)$ & $-0.0995(8)$ \\
\hline$N(2)$ & $1.0515(8)$ & $0.7304(4)$ & $0.0613(5)$ & $\mathrm{C}(29)$ & $0.7911(12)$ & $0.8661(6)$ & $-0.0558(8)$ \\
\hline $\mathrm{N}(3)$ & $0.8907(9)$ & $0.7488(5)$ & $0.1426(5)$ & $C(30)$ & $0.7464(11)$ & $0.8100(5)$ & $-0.0390(7)$ \\
\hline$N(4)$ & $0.8109(8)$ & $0.6467(5)$ & $0.1872(6)$ & $\mathrm{C}(31)$ & $0.6511(11)$ & $0.8010(5)$ & $0.0048(7)$ \\
\hline$N(5)$ & $0.8505(9)$ & $0.6526(4)$ & $-0.1147(5)$ & $C(32)$ & $0.5897(12)$ & $0.8485(6)$ & $0.0311(8)$ \\
\hline$N(6)$ & $0.7890(8)$ & $0.7574(4)$ & $-0.0642(5)$ & $C(33)$ & $0.5024(12)$ & $0.8366(7)$ & $0.0746(9)$ \\
\hline$N(7)$ & $0.6275(8)$ & $0.7445(4)$ & $0.0194(6)$ & $C(34)$ & $0.4766(11)$ & $0.7782(6)$ & $0.0888(8)$ \\
\hline $\mathrm{N}(8)$ & $0.5874(8)$ & $0.6313(4)$ & $0.0362(5)$ & $C(35)$ & $0.5392(10)$ & $0.7310(6)$ & $0.0602(7)$ \\
\hline$C(1)$ & $1.0954(11)$ & $0.5554(6)$ & $0.0325(7)$ & $C(36)$ & $0.5175(10)$ & $0.6668(5)$ & $0.0700(6)$ \\
\hline $\mathrm{C}(2)$ & $1.1707(11)$ & $0.5288(6)$ & $-0.0104(7)$ & $C(37)$ & $0.4369(11)$ & $0.6451(6)$ & $0.1109(7)$ \\
\hline $\mathrm{C}(3)$ & $1.2373(11)$ & $0.568 \mathrm{l}(6)$ & $-0.0488(7)$ & $\mathrm{C}(38)$ & $0.4253(12)$ & $0.5836(7)$ & $0.1199(7)$ \\
\hline$C(4)$ & $1.2311(11)$ & $0.6292(6)$ & $-0.0409(7)$ & $C(39)$ & $0.4986(12)$ & $0.5455(6)$ & $0.0867(7)$ \\
\hline$C(5)$ & $1.1522(10)$ & $0.6501(5)$ & $0.0053(7)$ & $\mathrm{C}(40)$ & $0.5745(11)$ & $0.5728(6)$ & $0.0457(7)$ \\
\hline$C(6)$ & $1.1360(11)$ & $0.7169(6)$ & $0.0190(7)$ & $O(11)$ & $0.2701(10)$ & $0.7540(6)$ & $0.2163(6)$ \\
\hline$C(7)$ & $1.2021(12)$ & $0.7608(6)$ & $-0.0122(8)$ & $O(12)$ & $0.6195(10)$ & $0.2405(6)$ & $0.1404(6)$ \\
\hline $\mathrm{C}(8)$ & $1.1814(13)$ & $0.8205(6)$ & $0.0047(9)$ & $O(13)$ & $0.2914(10)$ & $0.8804(6)$ & $0.2182(7)$ \\
\hline$C(9)$ & $1.0949(13)$ & $0.8351(7)$ & $0.0493(9)$ & $O(14)$ & $0.6307(15)$ & $0.9917(6)$ & $0.2099(7)$ \\
\hline$C(10)$ & $1.0295(12)$ & $0.7883(5)$ & $0.0767(7)$ & $O(15)$ & $0.6361(13)$ & $0.1164(7)$ & $0.1341(9)$ \\
\hline$C(11)$ & $0.9363(12)$ & $0.7992(6)$ & $0.1194(7)$ & $O(16)$ & $0.265(3)$ & $0.9721(8)$ & $0.0957(16)$ \\
\hline $\mathrm{C}(12)$ & $0.8968(14)$ & $0.8566(6)$ & $0.1357(8)$ & $O(17)$ & $0.882(4)$ & $0.0069(9)$ & $0.0968(16)$ \\
\hline$C(13)$ & $0.8003(14)$ & $0.8583(7)$ & $0.1764(9)$ & $O(18)$ & $0.500(3)$ & $0.0160(11)$ & $0.0880(15)$ \\
\hline$C(14)$ & $0.7585(14)$ & $0.8083(7)$ & $0.2008(9)$ & $O(19)$ & 0 & 0 & 0 \\
\hline
\end{tabular}

Table 4 Non-hydrogen atomic coordinates with e.s.d.s for $\left[\{\mathrm{FeL}(\mathrm{Cl})\}_{2}(\mu-\mathrm{O})\right]$

\begin{tabular}{llll} 
Atom & $x$ & $y$ & $z$ \\
$\mathrm{Fe}$ & $0.56420(9)$ & 0 & $0.37896(13)$ \\
$\mathrm{Cl}(1)$ & $0.69614(16)$ & 0 & $0.30625(24)$ \\
$\mathrm{O}(1)$ & $\frac{1}{2}$ & 0 & $\frac{1}{2}$ \\
$\mathrm{~N}(1)$ & $0.6010(3)$ & $0.1491(4)$ & $0.4432(5)$ \\
$\mathrm{N}(2)$ & $0.4900(3)$ & $0.0928(4)$ & $0.2286(5)$ \\
$\mathrm{C}(1)$ & $0.6572(4)$ & $0.1718(5)$ & $0.5591(7)$ \\
$\mathrm{C}(2)$ & $0.6747(5)$ & $0.2669(6)$ & $0.5997(8)$ \\
$\mathrm{C}(3)$ & $0.6340(6)$ & $0.3400(6)$ & $0.5192(9)$ \\
$\mathrm{C}(4)$ & $0.5764(5)$ & $0.3172(5)$ & $0.4007(8)$ \\
$\mathrm{C}(5)$ & $0.5602(4)$ & $0.2223(5)$ & $0.3648(7)$ \\
$\mathrm{C}(6)$ & $0.4978(4)$ & $0.1884(5)$ & $0.2425(7)$ \\
$\mathrm{C}(7)$ & $0.4491(5)$ & $0.2507(5)$ & $0.1467(7)$ \\
$\mathrm{C}(8)$ & $0.3914(5)$ & $0.2107(6)$ & $0.0362(7)$ \\
$\mathrm{C}(9)$ & $0.3835(5)$ & $0.1117(6)$ & $0.0236(7)$ \\
$\mathrm{C}(10)$ & $0.4339(4)$ & $0.0537(4)$ & $0.1218(7)$ \\
$\mathrm{Cl}(2)$ & $0.1702(3)$ & 0 & $0.1360(4)$ \\
$\mathrm{O}(2)$ & $0.2261(15)$ & 0 & $0.2428(17)$ \\
$\mathrm{O}(3)$ & $0.1922(10)$ & 0 & $0.0198(20)$ \\
$\mathrm{O}(4)$ & $0.1308(12)$ & $0.0727(10)$ & $0.1306(13)$ \\
$\mathrm{O}(5)$ & $0.9333(9)$ & 0 & $0.2244(10)$ \\
\hline
\end{tabular}

product, the crystal structure of which has been determined, ${ }^{11}$ which slowly converts to $\left[\left\{\mathrm{FeL}(\mathrm{Cl})_{2}\right\}(\mu-\mathrm{O})\right]^{2+} 3$ in acetonitrileethanol solution.

The first unequivocal evidence for the tetradentate coordination mode of $\mathrm{L}$ was the crystal-structure analysis of $\left[\mathrm{CoL}\left(\mathrm{OH}_{2}\right)\left(\mathrm{SO}_{3}\right)\right] \mathrm{NO}_{3} \cdot \mathrm{H}_{2} \mathrm{O},{ }^{12}$ followed by characterization of analogous complexes of copper(II), ${ }^{13}$ nickel(II), ${ }^{3 a}$ chromium(III), ${ }^{3 b}$ palladium(II), ${ }^{3 c}$ platinum(II), ${ }^{4 a}$ rhenium(V), ${ }^{4 b}$ tungsten(IV), ${ }^{4 c}$ ruthenium(II) ${ }^{4 d}$ and manganese(II). ${ }^{4 e}$ The

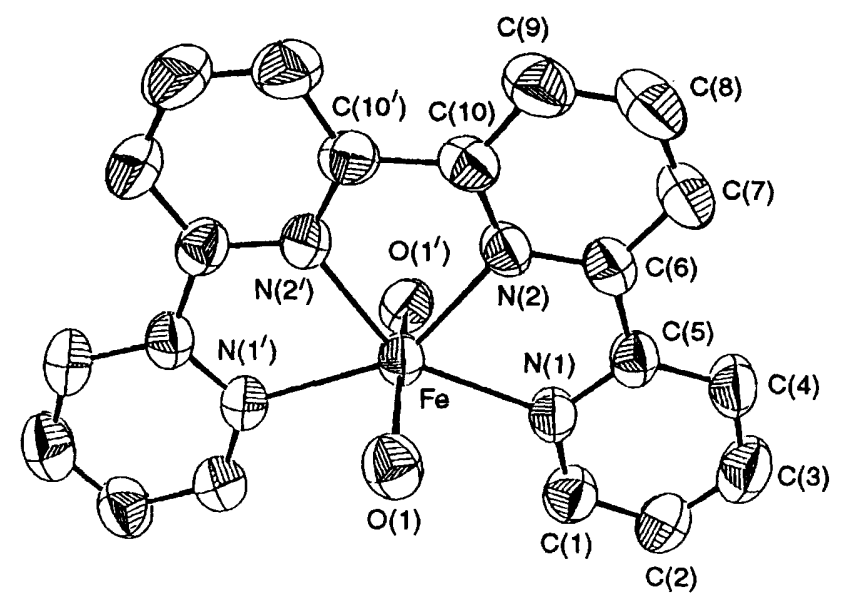

Fig. 1 Perspective view of $\left[\mathrm{FeL}\left(\mathrm{OH}_{2}\right)_{2}\right]^{2+} 1$; primed atoms are generated by a two-fold symmetry axis

molecular structure of $\left[\mathrm{FeL}\left(\mathrm{OH}_{2}\right)_{2}\right]^{2+}$ is shown in Fig. 1. It contains two mirror planes probably because of the coplanarity of the quaterpyridine ligand. The two axial $\mathrm{H}_{2} \mathrm{O}$ ligands are at a skew angle, pointing towards the $\mathrm{N}(1)-\mathrm{N}\left(1^{\prime}\right)$ axis between the two terminal pyridyl groups, leading to a $\mathrm{O}-\mathrm{Fe}-\mathrm{O}$ angle of $162.3(2)^{\circ}$. The $\mathrm{Fe}-\mathrm{OH}_{2}$ distance of $2.141(5) \AA$ is normal and is comparable to the average value of 2.123(6) $\AA$ observed in the high-spin complex $\left[\mathrm{Fe}\left(\mathrm{H}_{2} \mathrm{O}\right)_{6}\right]^{2+} .{ }^{14}$

A perspective view of $\mathbf{2}$ is shown in Fig. 2. The structure features an interesting and rare example of an $\mathrm{Fe}^{\mathrm{ll}}$ complex with a $O, O^{\prime}$-co-odinating $\left[\mathrm{ClO}_{4}\right]^{-}$ion. Iron complexes containing singly bound perchlorate ligands are not uncommon and have 
Table 5 Selected bond lengths $(\AA)$ and angles $\left(^{\circ}\right)$ for the iron complexes

Complex 1

\begin{tabular}{|c|c|c|c|c|c|c|c|}
\hline $\begin{array}{l}\mathrm{Fe}-\mathrm{O} \\
\mathrm{C}(5)-\mathrm{C}(6)\end{array}$ & $\begin{array}{l}2.141(5) \\
1.46(1)\end{array}$ & $\begin{array}{l}\mathrm{Fe}-\mathrm{N}(1) \\
\mathrm{C}(10)-\mathrm{C}\left(10^{\prime}\right)\end{array}$ & $\begin{array}{l}2.198(5) \\
1.49(1)\end{array}$ & $\mathrm{Fe}-\mathrm{N}(2)$ & $2.138(5)$ & & \\
\hline $\begin{array}{l}\mathrm{O}(1)-\mathrm{Fe}-\mathrm{O}\left(1^{\prime}\right) \\
\mathrm{N}(1)-\mathrm{Fe}-\mathrm{N}\left(2^{\prime}\right) \\
\mathrm{N}(1)-\mathrm{C}(5)-\mathrm{C}(6)\end{array}$ & $\begin{array}{l}162.3(2) \\
147.3(2) \\
115.9(5)\end{array}$ & $\begin{array}{l}\mathrm{N}(1)-\mathrm{Fe}-\mathrm{N}\left(1^{\prime}\right) \\
\mathrm{N}(1)-\mathrm{Fe}-\mathrm{O}(1) \\
\mathrm{N}(2)-\mathrm{C}(10)-\mathrm{C}(10)\end{array}$ & $\begin{array}{r}138.6(2) \\
86.3(2) \\
113.3(6)\end{array}$ & $\begin{array}{l}\mathrm{N}(1)-\mathrm{Fe}-\mathrm{N}(2) \\
\mathrm{N}(1)-\mathrm{Fe}-\mathrm{O}\left(1^{\prime}\right)\end{array}$ & $\begin{array}{l}74.1(2) \\
87.5(2)\end{array}$ & $\begin{array}{l}\mathrm{N}(1)-\mathrm{Fe}-\mathrm{O}(1) \\
\mathrm{N}(2)-\mathrm{Fe}-\mathrm{O}\left(1^{\prime}\right)\end{array}$ & $\begin{array}{l}97.0(2) \\
97.2(2)\end{array}$ \\
\hline $\begin{array}{l}\text { Complex } 2 \\
\mathrm{Fe}(1)-\mathrm{O} \\
\mathrm{Fe}(1)-\mathrm{N}(1) \\
\mathrm{Fe}(2)-\mathrm{O} \\
\mathrm{Fe}(2)-\mathrm{N}(5) \\
\mathrm{C}(5)-\mathrm{C}(6) \\
\mathrm{C}(25)-\mathrm{C}(26)\end{array}$ & $\begin{array}{l}1.797(8) \\
2.31(1) \\
1.785(8) \\
2.30(2) \\
1.51(2) \\
1.46(2)\end{array}$ & $\begin{array}{l}\mathrm{Fe}(1)-\mathrm{O}(1) \\
\mathrm{Fe}(1)-\mathrm{N}(2) \\
\mathrm{Fe}(2)-\mathrm{O}(5) \\
\mathrm{Fe}(2)-N(6) \\
\mathrm{C}(10)-\mathrm{C}(11) \\
\mathrm{C}(30)-\mathrm{C}(31)\end{array}$ & $\begin{array}{l}2.16(1) \\
2.21(1) \\
2.15(1) \\
2.20(2) \\
1.42(2) \\
1.45(2)\end{array}$ & $\begin{array}{l}\mathrm{Fe}(1)-\mathrm{O}(2) \\
\mathrm{Fe}(1)-\mathrm{N}(3) \\
\mathrm{Fe}(2)-\mathrm{O}(6) \\
\mathrm{Fe}(2)-\mathrm{N}(7) \\
\mathrm{C}(15)-\mathrm{C}(16) \\
\mathrm{C}(35)-\mathrm{C}(36)\end{array}$ & $\begin{array}{l}2.14(1) \\
2.20(1) \\
2.18(1) \\
2.24(2) \\
1.45(2) \\
1.45(2)\end{array}$ & $\begin{array}{l}F e(1)-N(4) \\
F e(2)-N(8)\end{array}$ & $\begin{array}{l}2.29(1) \\
2.25(2)\end{array}$ \\
\hline $\begin{array}{l}\mathrm{Fe}(1)-\mathrm{O}-\mathrm{Fe}(2) \\
\mathrm{O}-\mathrm{Fe}(1)-\mathrm{N}(3) \\
\mathrm{N}(1)-\mathrm{Fe}(1)-\mathrm{N}(2) \\
\mathrm{N}(1)-\mathrm{Fe}(1)-\mathrm{N}(4) \\
\mathrm{O}-\mathrm{Fe}(2)-\mathrm{N}(5) \\
\mathrm{N}(5)-\mathrm{Fe}(2)-\mathrm{N}(6) \\
\mathrm{N}(5)-\mathrm{Fe}(2)-\mathrm{N}(8) \\
\mathrm{N}(1)-\mathrm{C}(5)-\mathrm{C}(6) \\
\mathrm{N}(4)-\mathrm{C}(16)-\mathrm{C}(15)\end{array}$ & $\begin{array}{c}155.2(4) \\
96.1(4) \\
70.1(3) \\
148.3(4) \\
86.1(4) \\
71.2(3) \\
148.6(3) \\
112.5(10) \\
113.7(12)\end{array}$ & $\begin{array}{l}\mathrm{O}-\mathrm{Fe}(1)-\mathrm{N}(1) \\
\mathrm{O}-\mathrm{Fe}(1)-\mathrm{N}(4) \\
\mathrm{N}(1)-\mathrm{Fe}(1)-\mathrm{N}(3) \\
\mathrm{N}(2)-\mathrm{Fe}(1)-\mathrm{N}(3) \\
\mathrm{O}-\mathrm{Fe}(2)-\mathrm{N}(6) \\
\mathrm{N}(5)-\mathrm{Fe}(2)-\mathrm{N}(7) \\
\mathrm{N}(6)-\mathrm{Fe}(2)-\mathrm{N}(7) \\
\mathrm{N}(2)-\mathrm{C}(10)-\mathrm{C}(11) \\
\mathrm{N}(5)-\mathrm{C}(25)-\mathrm{C}(26)\end{array}$ & $\begin{array}{r}90.5(4) \\
86.0(4) \\
140.8(4) \\
70.7(4) \\
99.5(3) \\
141.7(4) \\
70.7(3) \\
117.0(11) \\
116.9(10)\end{array}$ & $\begin{array}{l}\mathrm{N}(2)-\mathrm{Fe}(1)-\mathrm{N}(4) \\
\mathrm{O}-\mathrm{Fe}(2)-\mathrm{N}(7) \\
\mathrm{N}(6)-\mathrm{Fe}(2)-\mathrm{N}(8) \\
\\
\mathrm{N}(3)-\mathrm{C}(15)-\mathrm{C}(16) \\
\mathrm{N}(6)-\mathrm{C}(26)-\mathrm{C}(25)\end{array}$ & $\begin{array}{l}141.5(4) \\
103.3(4) \\
140.2(3) \\
115.9(11) \\
114.6(10)\end{array}$ & $\begin{array}{l}\mathrm{N}(3)-\mathrm{Fe}(1)-\mathrm{N}(4) \\
\mathrm{O}-\mathrm{Fe}(2)-\mathrm{N}(8) \\
\mathrm{N}(7)-\mathrm{Fe}(2)-\mathrm{N}(8) \\
\\
\mathrm{N}(7)-\mathrm{C}(35)-\mathrm{C}(36) \\
\mathrm{N}(8)-\mathrm{C}(36)-\mathrm{C}(35)\end{array}$ & $\begin{array}{l}70.8(4) \\
88.1(4) \\
69.6(3) \\
115.5(10) \\
113.1(10)\end{array}$ \\
\hline $\begin{array}{l}\text { Complex } 3 \\
\text { Fe-O } \\
\text { C }(5)-C(6)\end{array}$ & $\begin{array}{l}1.779(1) \\
1.48(1)\end{array}$ & $\begin{array}{l}\mathrm{Fe}-\mathrm{Cl} \\
\mathrm{C}(10)-\mathrm{C}\left(10^{\prime}\right)\end{array}$ & $\begin{array}{l}2.348(3) \\
1.48(1)\end{array}$ & $\mathrm{Fe}-\mathrm{N}(1)$ & $2.191(5)$ & $\mathrm{Fe}-\mathrm{N}(2)$ & $2.129(5)$ \\
\hline $\begin{array}{l}\mathrm{O}-\mathrm{Fe}-\mathrm{Cl} \\
\mathrm{N}(2)-\mathrm{Fe}-\mathrm{N}(2) \\
\mathrm{N}(2)-\mathrm{Fe}-\mathrm{Cl}\end{array}$ & $\begin{array}{c}154.95(9) \\
73.7(2) \\
97.4(2)\end{array}$ & $\begin{array}{l}\mathrm{Fe}-\mathrm{O}-\mathrm{Fe}^{\prime} \\
\mathrm{N}(1)-\mathrm{Fe}-\mathrm{O} \\
\mathrm{N}(1)-\mathrm{C}(5)-\mathrm{C}(6)\end{array}$ & $\begin{array}{l}180.0 \\
86.3(1) \\
113.4(6)\end{array}$ & $\begin{array}{l}\mathrm{N}(1)-\mathrm{Fe}-\mathrm{N}\left(1^{\prime}\right) \\
\mathrm{N}(2)-\mathrm{Fe}-\mathrm{O} \\
\mathrm{N}(2)-\mathrm{C}(10)-\mathrm{C}\left(10^{\prime}\right)\end{array}$ & $\begin{array}{l}139.0(2) \\
102.6(1) \\
113.5(6)\end{array}$ & $\begin{array}{l}\mathrm{N}(1)-\mathrm{Fe}-\mathrm{N}(2) \\
\mathrm{N}(1)-\mathrm{Fe}-\mathrm{Cl}\end{array}$ & $\begin{array}{l}85.0(1) \\
85.0(1)\end{array}$ \\
\hline
\end{tabular}

Table 6 Energy and composition of the near-frontier orbitals of the cation of complex 2

\begin{tabular}{|c|c|c|c|c|}
\hline \multirow[b]{2}{*}{ Energy/eV } & \multicolumn{4}{|c|}{ Composition $(\%)$} \\
\hline & $2 \mathrm{Fe}$ & $\mathrm{O}$ & $2 \mathrm{ClO}_{4}$ & $2 \mathrm{~L}$ \\
\hline 1.2625 & 0.34 & 0.06 & 0.06 & 99.54 \\
\hline 1.1537 & 0.47 & 0.19 & 0.11 & 99.23 \\
\hline 1.0694 & 0.71 & 0.12 & 0.19 & 98.98 \\
\hline 1.0040 & 0.74 & 0.39 & 0.25 & 98.62 \\
\hline 0.8789 & 0.31 & 0.10 & 0.05 & 99.54 \\
\hline 0.5306 (LUMO) & 0.57 & 0.38 & 0.18 & 98.87 \\
\hline-7.3385 (HOMO) & 51.68 & 42.89 & 3.07 & 2.36 \\
\hline-7.4691 (NHOMO) & 49.44 & 45.61 & 1.03 & 3.92 \\
\hline-8.4814 & 78.06 & 12.52 & 5.14 & 4.28 \\
\hline-8.7589 & 84.69 & 0.55 & 5.13 & 9.63 \\
\hline-8.9222 & 81.74 & 8.11 & 1.73 & 8.42 \\
\hline-9.5453 & 96.77 & 0.03 & 1.00 & 2.20 \\
\hline-11.2731 & 39.68 & 48.19 & 6.24 & 5.88 \\
\hline-11.4772 & 44.14 & 39.13 & 2.94 & 13.79 \\
\hline-11.6241 & 42.11 & 39.25 & 10.61 & 8.02 \\
\hline-12.0976 & 2.87 & 2.13 & 93.66 & 1.34 \\
\hline-12.1982 & 1.99 & 2.08 & 94.77 & 1.16 \\
\hline-12.3098 & 0.90 & 0.46 & 1.20 & 97.44 \\
\hline
\end{tabular}

been reviewed. ${ }^{15}$ Here, the iron atom is seven-co-ordinate and this is quite different from the co-ordination in $\mathbf{1}$ and in $\mathbf{3}$. The co-ordinated $\mathrm{Cl}-\mathrm{O}$ distances, ranging from $1.458(9)$ to $1.523(10) \AA$, are longer than all the unco-ordinated distances in 2 and 3 which may suggest the weakening of the $\mathrm{Cl}-\mathrm{O}$ bond through co-ordination of $\mathrm{ClO}_{4}^{-}$to $\mathrm{Fe}^{\mathrm{Il}}$.

Fig. 3 shows a perspective view of the complex cation of $\mathbf{3}$, which is unique among other iron complexes having $\alpha$-diimine ligands. For the $\left[\left\{\mathrm{Fe}(\mathrm{phen})_{2}\left(\mathrm{OH}_{2}\right)\right\}_{2} \mathrm{O}\right]^{4+}{ }^{16}\left[\left\{\mathrm{Fe}(\mathrm{phen})_{2} \mathrm{Cl}\right\}_{2^{-}}\right.$ $\mathrm{O}]^{2+17}$ and $\left[\left\{\mathrm{Fe}(\text { phen })\left(\mathrm{OH}_{2}\right)_{3}\right\}_{2} \mathrm{O}\right]^{4+18}$ (phen $=1,10$-phenanthroline) complexes studied previously, the two $\alpha$-diimine
Table 7 Energy and composition of the near-frontier orbitals of the cation of complex 3

\begin{tabular}{lrrrr} 
& \multicolumn{5}{l}{ Composition $(\%)$} \\
\cline { 2 - 5 } Energy/eV & $2 \mathrm{Fe}$ & \multicolumn{1}{l}{$\mathrm{O}$} & \multicolumn{1}{l}{$2 \mathrm{Cl}$} & \multicolumn{1}{l}{$2 \mathrm{~L}$} \\
0.7597 & 0.26 & 0.00 & 0.02 & 99.72 \\
0.6916 & 0.39 & 0.00 & 0.28 & 99.33 \\
0.6508 & 1.83 & 0.00 & 0.21 & 97.96 \\
0.6263 & 0.16 & 0.03 & 0.02 & 99.79 \\
0.2862 & 1.86 & 0.02 & 1.28 & 96.84 \\
0.0359 (LUMO) & 13.25 & 0.28 & 3.37 & 83.10 \\
-4.5653 (HOMO) & 77.97 & 2.20 & 18.34 & 1.49 \\
-4.9381 (NHOMO) & 72.30 & 1.79 & 24.16 & 1.75 \\
-10.0264 & 16.55 & 12.54 & 68.50 & 2.41 \\
-10.4536 & 23.05 & 8.42 & 66.50 & 2.03 \\
-12.6603 & 9.53 & 8.07 & 31.55 & 50.85 \\
-12.6902 & 4.42 & 1.87 & 71.42 & 22.29 \\
-12.7637 & 4.00 & 1.27 & 70.45 & 24.28 \\
-12.7800 & 4.65 & 3.24 & 35.12 & 56.99 \\
-12.9324 & 14.38 & 21.70 & 37.04 & 26.88 \\
-13.0930 & 10.42 & 16.00 & 24.18 & 49.41 \\
-13.0984 & 3.56 & 4.00 & 10.24 & 82.11 \\
-13.3623 & 13.34 & 13.87 & 32.43 & 40.36 \\
\hline
\end{tabular}

moieties are $c i s$ to each other and the $\mathrm{Fe}_{2} \mathrm{O}$ unit $\left(155-162^{\circ}\right)$ is bent, whereas in 3 , the two quaterpyridine planes are parallel and the $\mathrm{Fe}-\mathrm{O}-\mathrm{Fe}$ angle is $180^{\circ}$. Iron(III) complexes bearing a linear $\mathrm{Fe}-\mathrm{O}-\mathrm{Fe}$ unit have been reported previously: the observed $\mathrm{Fe}-\mathrm{O}$ distance of $1.779(1) \AA$ in 3 is comparable to that of $1.772-1.773$ in $\left[\left\{\mathrm{Fe}\left(\mathrm{H}_{2} \mathrm{O}\right)_{2}\left(\mathrm{~L}^{\prime}\right)\right\}_{2} \mathrm{O}\right]\left(\mathrm{L}^{\prime}=4\right.$-chloro2,6-pyridinedicarboxylate $),{ }^{19}$ and of $1.763 \AA$ in $\left[\left(\mathrm{FeL}^{\prime \prime}\right)_{2} \mathrm{O}\right]$ $\left(\mathrm{H}_{2} \mathrm{~L}^{\prime \prime}=5,10,15,20\right.$-tetraphenylporphyrin $){ }^{20}$ The two quaterpyridines in 3 are coplanar and are separated by about $3.56 \AA$.

Complexes 1 and 2 are diamagnetic. Complex 3 is paramagnetic with a measured $\mu_{\text {eff }}$ of $1.75 \mu_{B}$ per iron centre. 


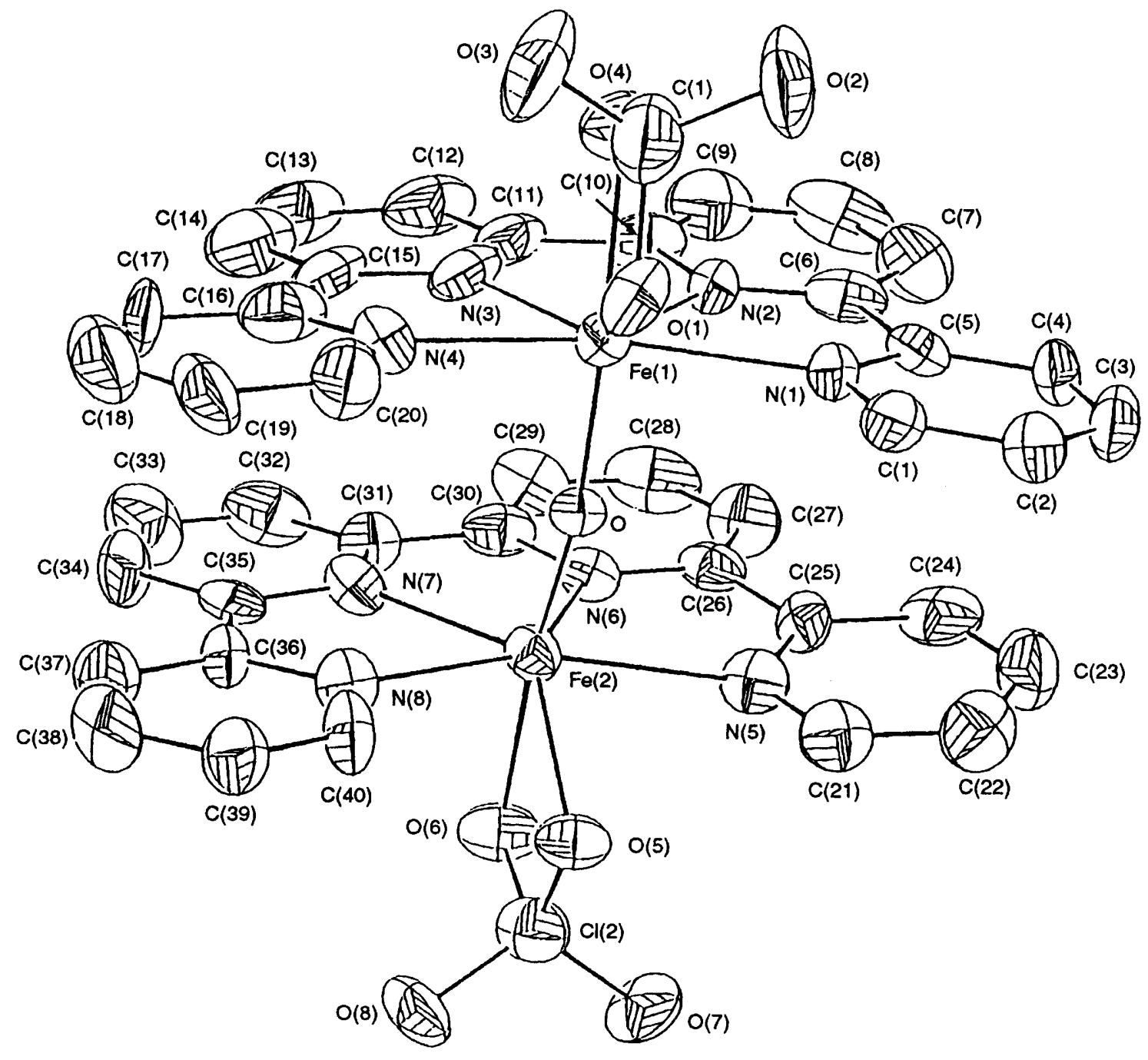

Fig. 2 Perspective view of $\left[\left\{\mathrm{FeL}\left(\mathrm{ClO}_{4}\right)\right\}_{2}(\mu-\mathrm{O})\right] 2$

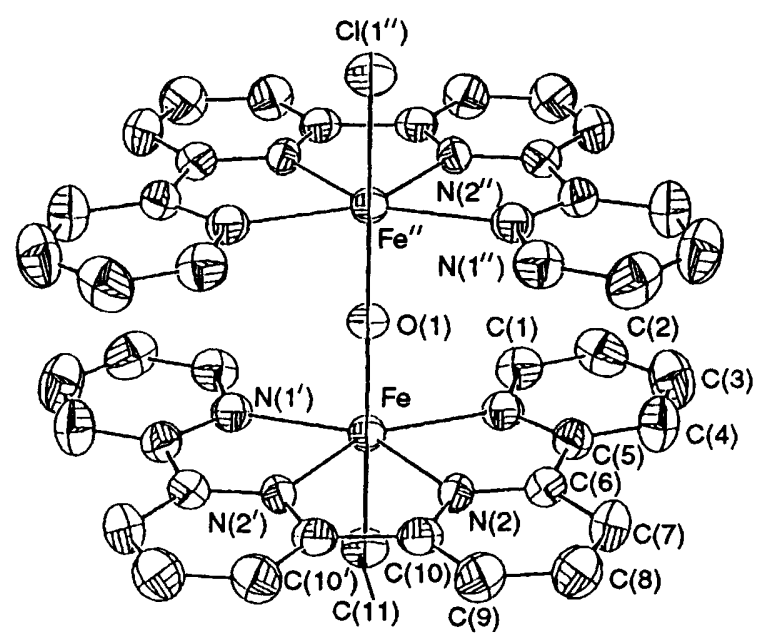

Fig. 3 Perspective view of $[\{\mathrm{FeL}(\mathrm{Cl})\}(\mu-\mathrm{O})]^{2+}$ 3; primed atoms are generated by a two-fold mirror plane

This is in line with the results of the calculations described below, which indicates an open-shell $(S=1)$ ground state. Molecular-orbital calculations on 2 and 3 have been undertaken and the energies and composition of the near-frontier orbitals are listed in Tables 6 and 7 respectively.

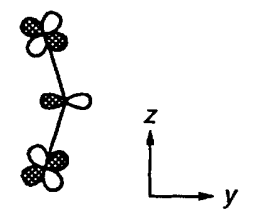

Fig. 4 The HOMO of the complex cation 2

The highest occupied molecular orbital (HOMO) of 2 is shown in Fig. 4. In the HOMO, however, the oxygen $p_{y}$ orbital is displaced from the $\mathrm{Fe}-\mathrm{Fe}$ axis. This results in a decrease in the antibonding interaction between the $\mathrm{O}\left(\mathrm{p}_{y}\right)$ and two $\mathrm{Fe}\left(\mathrm{d}_{\mathrm{yz}}\right)$ orbitals. As a result, the two $\mathrm{Fe}-\mathrm{L}$ planes are tilted. In the next HOMO (NHOMO), the bending of $\mathrm{Fe}-\mathrm{O}-\mathrm{Fe}$ has a small influence in the orbital energy as the two $d_{x z}(F e)$ and $p_{x}(O)$ orbitals are not in the same plane. The LUMO is mainly comprised of the $\pi^{*}$ orbital of $\mathrm{L}$. Thus, the intense low energy absorption band in the visible region can be ascribed to metalto-ligand charge-transfer transitions.

The total electron energy of 3 was calculated for both the open- $(S=1)$ and closed-shell $(S=0)$ states. The results showed that the former is more stable than the latter by 49.3 kcal $\mathrm{mol}^{-1}$ and that the HOMO and NHOMO have comparable energies. This is in accord with the experimental finding that complex 3 has two unpaired electrons The interaction between $\mathrm{Fe}\left(\mathrm{d}_{x z}, \mathrm{~d}_{y z}\right)$ and $\mathrm{Cl}\left(\mathrm{p}_{x}, \mathrm{p}_{y}\right)$ orbitals is dominant in both the HOMO and NHOMO, and the two unpaired electrons are localized in the $\mathrm{Fe}-\mathrm{Cl}$ bonding 
orbitals. The LUMO is mainly composed of the $\pi^{*}$ orbital of $L$.

\section{Acknowledgements}

We acknowledge support from The Hong Kong Research Grants Council and The University of Hong Kong. C.-W. C. is thankful to the award of a Hung Hing-Ying Scholarship (199293) administrated by The University of Hong Kong.

\section{References}

1 V. Balzani, F. Bolleta, T. Gandolfi and M. Maestri, Top. Curr. Chem., 1978, 75, 1 .

2 E. C. Constable, Adv. Inorg. Chem., 1986, 30, 69; Prog. Inorg. Chem. 1994, 42, 67.

3 (a) E. C. Constable, S. M. Elder, J. Healy and D. A. Tocher, J. Chem. Soc., Dalton Trans., 1990, 1669; (b) E. C. Constable, S. M. Elder and D. A. Tocher, Polyhedron, 1992, 11, 1337; (c) E. C. Constable, S. M. Elder, J. Healy and M. D. Ward, J. Am. Chem. Soc., 1990, 112 4590.

4 (a) C. W. Chan, C. M. Che, M. C. Cheng and Y. Wang, Inorg. Chem., 1992, 31, 4874, (b) C. M. Che, Y. P. Wang, K. S. Yeung, K. Y. Wong and S. M. Peng, J. Chem. Soc., Dalton Trans., 1992, 2675; (c) S. M. Yang, K. K. Cheung and C. M. Che, J. Chem Soc., Dalton Trans., 1993, 3515; (d) C. W. Chan, T. F. Lai and C. M. Che, J. Chem. Soc., Dalton Trans., 1994, 895; (e) C. W. Chan, C. M. Che and S. M. Peng, Polyhedron, 1993, 12, 2169.

5 M. Branca, B. Pispisa and C. Aurisicchio, J. Chem. Soc, Dalton Trans., 1976, 1543; M. Cerdonio, F. Mogno, B. Pispisa, G. L. Romani and S. Vitale, Inorg. Chem., 1977, 16, 400; B. Pispisa, M. Barteri and M. Farinella, Inorg. Chem., 1983, 22, 3166;
B. Pispisa, A. Palleschi and G. Paradossi, J. Mol. Catal., 1987, 42, 269.

6 D. H. R. Barton and D. Doller, Acc. Chem. Res., 1992, 25, 504; T. Kojima, R. A. Leising, S. Yan and L. Que, J. Am. Chem. Soc., $1993,115,11328$.

7 NRCVAX, E. J. Cabe, Y. Le Page, J. P. Charland, F. L. Lee and P. S. White, J. Appl. Crystallogr., 1989, 22, 384.

8 A. Earnshaw, Magnetochemistry, Academic Press, New York, 1969

9 G. C. Allen and D. W. Clark, J. Chem. Soc. A., 1970, 2668.

10 J. A. Pople and D. L. Beveridge, Approximate Molecular Orbital Theory, McGraw-Hill, New York, 1970.

11 C. Y. Lee and C. M. Che, unpublished work.

12 E. N. Maslen, C. L. Raston and A. H. White, J. Chem. Soc, Dalton Trans., 1975, 323.

13 W. Henke, S. Kremer and D. Reinen, Z. Anorg. Allg. Chem., 1982, 491,124

14 J. Strouse, S. W. Layten and C. E. Strouse, J. Am. Chem. Soc., 1977, $99,562$.

15 N. M. N. Gowda, S. B. Naikar and G. K. N. Reddy, Adv. Inorg. Chem. Radiochem., 1984, 28, 255.

16 J. E. Plowman, T. M. Loehr, C. K. Schauer and O. P. Anderson, Inorg. Chem., 1984, 23, 3553.

17 P. C. Healy, B. W. Skelton and A. H. White, Aust. J. Chem., 1983, 36, 2057.

18 P. C. Healy, J. M. Patrick and A. H. White, Aust. J. Chem., 1984, 37, 1405.

19 C. C. Ou, R. G. Wollmann, D. N. Hendrickson, J. A. Potenza and H. J. Schugar, J. Am. Chem. Soc., 1978, 100, 4717.

20 A. B. Hoffman, D. M. Collins, V. M. Day, E. B. Fleischer, T. S. Srivastava and J. L. Hoard, J. Am. Chem. Soc., 1972, 94, 3620.

Received 31st January 1995; Paper 5/00573F 